\title{
Tópicos Emergentes em Insuficiência Cardíaca: Inibidores do Cotransportador Sódio-Glicose 2 (iSGLT2) na IC
}

\author{
Emerging Topics in Heart Failure: Sodium-Glucose Co-Transporter 2 Inhibitors (SGLT2i) in HF
}

\author{
Edimar Alcides Bocchi, ${ }^{\circledR}$ Andréa Biolo, ${ }^{2,3,4}$ Lidia Zytynski Moura, ${ }^{5}{ }^{\circledR}$ José Albuquerque Figueiredo Neto, ${ }^{6}{ }^{\circledR}$ \\ Carlos Eduardo Lucena Montenegro, ${ }^{7 \bullet}$ Denilson Campos de Albuquerque ${ }^{8,9}{ }^{\circledR}$ \\ Clínica de Insuficiência Cardíaca do Instituto do Coração (Incor) da Universidade de São Paulo, ${ }^{1}$ São Paulo, SP - Brasil \\ Faculdade de Medicina da Universidade Federal do Rio Grande do Sul (UFRGS), ${ }^{2}$ Porto Alegre, RS - Brasil \\ Hospital de Clínicas de Porto Alegre (HCPA), ${ }^{3}$ Porto Alegre, RS - Brasil \\ Serviço de Cardiologia do Hospital Moinhos de Vento, ${ }^{4}$ Porto Alegre, $R S$ - Brasil \\ Pontifícia Universidade Católica do Paraná, ${ }^{5}$ Curitiba, PR - Brasil \\ Universidade Federal do Maranhão, ${ }^{6}$ São Luís, MA - Brasil \\ Pronto Socorro Cardiológico de Pernambuco (PROCAPE/UPE), 7 Pernambuco, RE - Brasil \\ Universidade do Estado do Rio de Janeiro, ${ }^{8}$ Rio de Janeiro, RJ - Brasil \\ Instituto D'Or de Pesquisa e Ensino, ${ }^{9}$ Rio de Janeiro, RJ - Brasil \\ Carta científica referente ao Heart Failure Summit Brazil 2020 / Departamento de Insuficiência Cardíaca - DEIC/SBC
}

\section{Possíveis mecanismos de ação}

Os inibidores do cotransportador de sódio-glicose-2 (SGLT2) inibem a reabsorção da glicose no túbulo contorcido proximal, resultando em glicosúria e redução dos níveis glicêmicos. Entretanto, esse efeito não parece explicar os benefícios dos ISGLT2 em pacientes com insuficiência cardíaca (IC). ${ }^{1,2}$

Seu benefício também parece não ser diretamente relacionado ao efeito nos fatores de risco cardiovasculares clássicos [hipertensão arterial sistêmica, diabetes melito (DM), dislipidemia], uma vez que a redução de desfechos no estudo EMPA-REG não foi dependente do perfil metabólico/hemodinâmico basal dos pacientes ou de sua variação ao longo do estudo. ${ }^{3}$

Entre os mecanismos mais aceitos para explicar o modo de ação dos ISGLT2 na IC, estão a melhora na tensão parietal do ventrículo esquerdo secundário à diminuição da pré (efeito da natriurese e diurese osmótica) e pós-carga (melhora na função endotelial e redução da pressão arterial). ${ }^{4-6}$ Mecanismos metabólicos incluem a melhora no metabolismo e bioenergética do cardiomiócito (maior cetogênese e aumento da oferta de $\beta$-hidroxibutirato), ${ }^{7}$ inibição da bomba sódio-hidrogênio miocárdica (o que leva a maior concentração de cálcio na mitocôndria), ${ }^{8}$ redução da necrose e fibrose cardíacas (inibição da síntese de colágeno) ${ }^{9}$ e alterações na produção de citocinas e no tecido gorduroso epicárdico. ${ }^{10}$

Entretanto, ainda existem dúvidas sobre a real contribuição desses mecanismos.

Os benefícios são observados com e sem DM, colocando em dúvida o papel da cetogênese.

\section{Palavras-chave}

insuficiência cardíaca; iSGLT2.

Correspondência: Edimar Alcides Bocchi •

Rua Dr. Melo Alves, 690, 4ํandar. CEP 01417-010, Cerqueira César, São

Paulo, SP - Brasil

E-mail: dcledimar@incor.usp.br

Artigo recebido em 12/01/2021, revisado em 25/01/2021, aceito em 25/01/2021

DOI: https://doi.org/10.36660/abc.20210031
O efeito diurético dos ISGLT2 não foi observado no DAPA-HF (seja potencializando diuréticos ou reduzindo níveis de peptídeos natriuréticos). ${ }^{11}$ Dessa forma, o melhor conhecimento dos principais mecanismos ainda depende de estudos em modelos experimentais e de outros em andamento, como EMPERORpreserved, EMPA-HEART e DELIVER.

Os possíveis mecanismos de ação dessa classe terapêutica estão sintetizados na Figura 1.

\section{Novas evidências na prevenção de IC}

Em 2015, foi publicado o primeiro grande estudo dessa classe terapêutica (EMPA-REG OUTCOME), ${ }^{12}$ que avaliou a empagliflozina em pacientes com DM2, doença cardiovascular estabelecida e recebendo tratamento usual. Entre os pacientes que receberam empagliflozina, houve redução significativa dos principais eventos cardiovasculares adversos [MACE = morte cardiovascular (CV), infarto do miocárdio (IM) não fatal ou acidente vascular cerebral (AVC) não fatal] [hazard ratio (HR): 0,86; intervalo de confiança (IC) 95\%: 0,74-0,99], e uma surpreendente redução de hospitalização por insuficiência cardíaca (HIC) (HR:0,65; IC 95\%: 0,50-0,85). O CANVASProgram, ${ }^{13}$ publicado em 2017, avaliou a canagliflozina em pacientes com DM2 com alto risco para eventos cardiovasculares e recebendo tratamento usual. Houve redução para o desfecho primário combinado (MACE $=$ morte $\mathrm{CV}$, IM não fatal ou $\mathrm{AVC}$ não fatal) e redução de $\mathrm{HIC}$ de $33 \%$ ( $H R=0,67$; IC 95\%: 0,520,87 ) bem como dos eventos renais combinados.

O estudo DECLARE-TIMI $58^{14}$ avaliou a dapagliflozina em pacientes com DM2 e doença aterosclerótica estabelecida ou múltiplos fatores de risco para doença aterosclerótica e recebendo tratamento usual. Não houve redução no desfecho primário combinado (MACE $=$ morte $\mathrm{CV}$, IM ou AVC). Foi observada redução para o desfecho combinado de mortalidade cardiovascular e HIC de 17\%, e uma redução de 27\% (HR: 0,73; IC 95\%: 0,61-0,88) para HIC. Mais recentemente o estudo Vertis$\mathrm{CV}^{15}$ avaliou a ertuglifozina (ainda não comercializada no Brasil) em pacientes com DM2, doença cardiovascular estabelecida e recebendo tratamento usual. Não houve redução no desfecho primário combinado (MACE $=$ morte CV, IM ou AVC). Foi observada, no entanto, redução de $30 \%$ na $\mathrm{HIC}$. 


\section{Carta Científica}

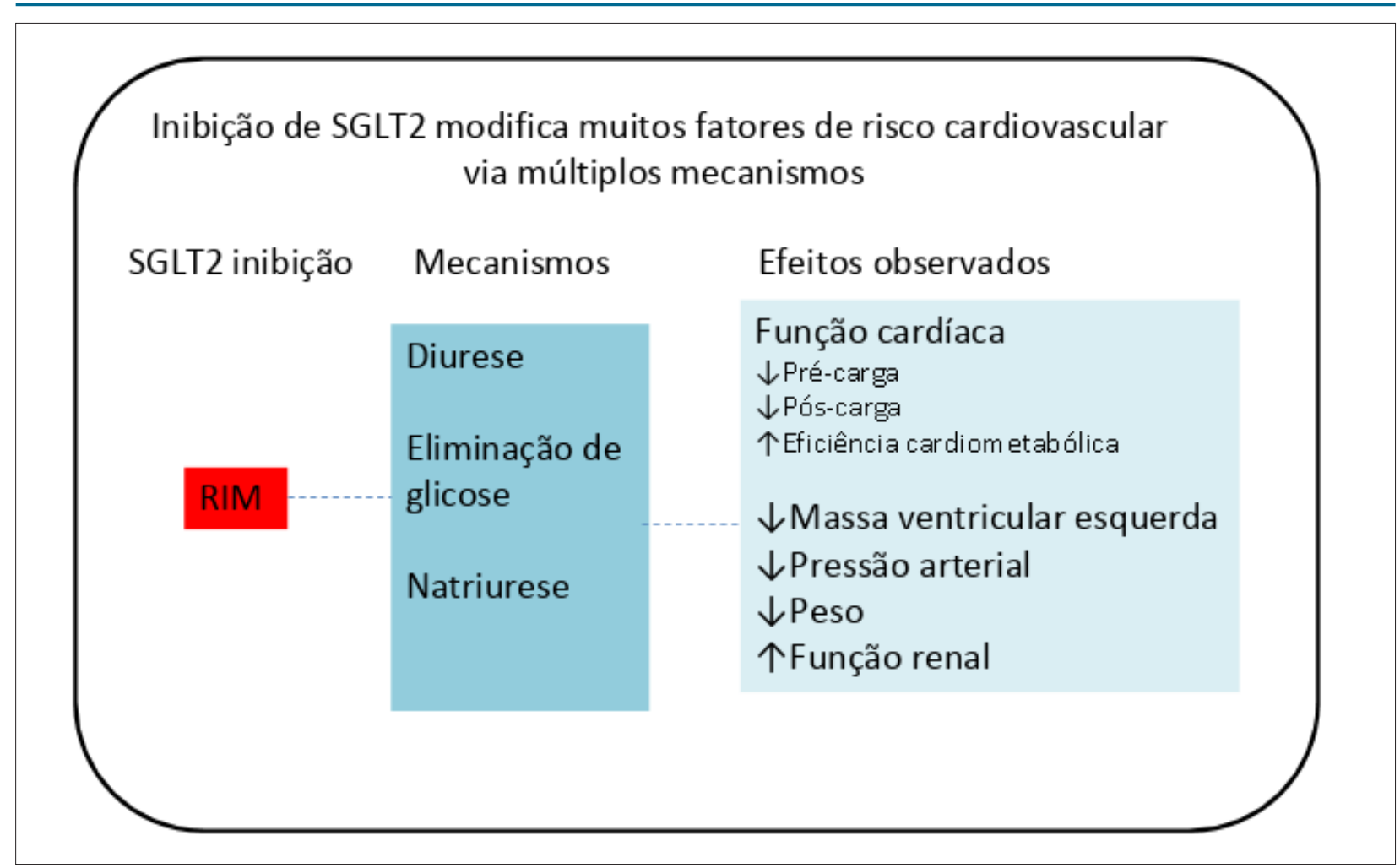

Figura 1 - Mecanismos de ação do iSGLT2. ISGLT2: inibidores do cotransportador de sódio-glicose-2.

Tomados em conjunto, os dados disponíveis demonstram a eficácia dos inibidores da SGLT2 na redução da incidência por IC em grupos de pacientes com DM2.

A avaliação de outros desfechos isoladamente evidenciou benefícios para o grupo que recebeu o medicamento. O HR foi de 0,73 (IC 95\%: 0,61-0,88) para hospitalização por IC; HR de 0,83 (IC 95\%: 0,73-0,95) para desfecho combinado de mortalidade CV e HIC; e HR de 0,53 (IC 95\%: 0,43-0,66) para insuficiência ou mortalidade renal.

Os resultados para o desfecho primário combinado (MACE $=$ morte CV, IM não fatal ou AVC não fatal) apontaram HR de 0,86 (IC 95\%: 0,74-0,99).

Novas evidências no tratamento da IC em pacientes com e sem DM

\section{Prevenção de IC no diabético}

A IC é sabidamente a segunda causa de doença cardiovascular no DM2. A prevalência de IC é de 9 a 22\%, o que representa quatro vezes a prevalência na população geral, sendo em geral maior no sexo feminino (redução de risco relativo (RRR) 1,95 vs 1,75$).{ }^{16}$ Dados recentes sugerem que o índice de massa corporal possui impacto maior no desenvolvimento de IC nos pacientes DM2 do que hemoglobina glicada por si só, diferente do desfecho IM/AVC. ${ }^{16}$

Assim sendo, os diferentes mecanismos das drogas hipoglicemiantes precisam ser considerados nesse desfecho de forma geral, e não apenas o controle glicêmico. ${ }^{17}$
Os inibidores de dipeptidil peptidase-4 (DPP-4) se mostraram neutros como classe em todos os aspectos da doença cardiovascular. Os agonistas do peptídeo semelhante a glucagon 1 (GLP1) como uma classe reduziram risco de doença cardiovascular aterosclerótica (redução de IM e/ou AVC). ${ }^{18}$

No entanto, foram os inibidores da SGLT2 que mostraram um benefício definitivo na redução da hospitalização por IC. ${ }^{19}$ Recentemente, o estudo DAPA HF e EMPEROR-Reduced demonstraram em pacientes com IC com fração de ejeção reduzida ampliação dos benefícios não somente nos diabéticos, como também em não diabéticos. Nesses estudos, foram encontradas redução de internações por IC, bem como redução de mortalidade cardiovascular com uso dos iSGLT2 como terapia de adição ao tratamento farmacológico otimizado para IC..$^{19}$

Assim, a metanálise de combinações demonstrou que um potencial regime ideal de tratamento com redução de desfechos CV e IC poderia ser a combinação de GLP1-a e SGLT2-i em um histórico de terapia com metformina. ${ }^{17,19}$

\section{iSGLT2 na ICFER - quais, para quem e em que momento}

O iSGLT2 (ertugliflozin) no estudo VERTIS CV reduziu hospitalização por IC em pacientes diabéticos com doença vascular por aterosclerose. ${ }^{15} \mathrm{O}$ iSGLT2 (empagliflozina) no estudo EMPEROR-Reduced reduziu o desfecho primário combinado de hospitalização por IC/morte CV; os desfechos secundários de hospitalização por IC e declínio da função renal; melhorou qualidade de vida, reduziu a hemoglobina glicada e o fragmento $\mathrm{N}$-terminal do peptídeo natriurético tipo $\mathrm{B}$ (NT-proBNP). ${ }^{20}$ 
A dapagliflozina, no estudo DAPA-HF, reduziu o desfecho combinado de hospitalização/visita urgente por IC e morte $\mathrm{CV}$; os desfechos secundários de morte cardiovascular/HIC, total de $\mathrm{HIC} /$ morte $\mathrm{CV}$; e morte de qualquer causa e melhorou qualidade de vida. ${ }^{21}$ Da mesma maneira, a dapaglifozina no estudo DECLARE-TIMI reduziu evento renal ${ }^{14} \mathrm{e}$, no DAPA$C K D$, reduziu o risco de sustentado declínio da função renal em pacientes com doença renal crônica, diabético ou não. ${ }^{7}$ Uma subanálise do estudo DAPA-HF demonstrou redução da progressão da função renal em pacientes com IC; ${ }^{8}$ uma análise preespecificada do EMPA-REG OUTCOME demonstrou que o ISGLT2 (empagliflozina) reduziu progressão da piora da função renal em diabéticos. ${ }^{3}$ No estudo CREDENCE, a canagliflozina reduziu a progressão da piora da função renal. ${ }^{22}$

A Figura 2 sintetiza os benefícios inquestionáveis na redução de hospitalização nos principais estudos.

A última Diretriz Brasileira de Insuficiência Cardíaca da SBC, coordenada pelo Departamento de Insuficiência Cardíaca (DEIC), foi publicada em 2018 e pouco conhecimento havia sobre o papel dos iSGLT2 no manuseio terapêutico da IC. ${ }^{23}$ Foi consenso no DEIC que era chegado o momento de revisitá-la. Para tanto, foram realizadas reuniões preparatórias, divisões de tópicos entre os diversos colaboradores e uma reunião virtual por conta da pandemia do COVID-19 que ocorreu em 04 de dezembro de 2020. Essa reunião teve a participação de ilustres especialistas na área de IC, atualizando, opinando e incluindo novas opções terapêuticas. Os iSGLT2 foram incorporados ao manuseio terapêutico da IC reunidos em uma tabela única que está sendo publicada dentro em breve.
Lista de Participantes do Heart Failure Summit Brazil 2020

/ Departamento de Insuficiência Cardíaca - DEIC/SBC

Aguinaldo Freitas Junior, Andréia Biolo, Antonio Carlos Pereira Barretto, Antônio Lagoeiro Jorge, Bruno Biselli, Carlos Eduardo Lucena Montenegro, Denilson Campos de Albuquerque, Dirceu Rodrigues de Almeida, Edimar Alcides Bocchi, Edval Gomes dos Santos Júnior, Estêvão Lanna Figueiredo, Evandro Tinoco Mesquita, Fabiana G. Marcondes-Braga, Fábio Fernandes, Fabio Serra Silveira, Felix José Alvarez Ramires, Fernando Atik, Fernando Bacal, Flávio de Souza Brito, Germano Emilio Conceição Souza, Gustavo Calado de Aguiar Ribeiro, Humberto Villacorta Jr., Jefferson Luis Vieira, João David de Souza Neto, João Manoel Rossi Neto, José Albuquerque de Figueiredo Neto, Lídia Ana Zytynski Moura, Livia Adams Goldraich, Luís Beck-da- Silva, Luís Eduardo Paim Rohde, Luiz Claudio Danzmann, Manoel Fernandes Canesin, Marcelo Bittencourt, Marcelo Westerlund Montera, Marcely Gimenes Bonatto, Marcus Vinicius Simões, Maria da Consolação Vieira Moreira, Miguel Morita Fernandes da Silva, Monica Samuel Avila, Mucio Tavares de Oliveira Junior, Nadine Clausell, Odilson Marcos Silvestre, Otavio Rizzi Coelho Filho, Pedro Vellosa Schwartzmann, Reinaldo Bulgarelli Bestetti, Ricardo Mourilhe Rocha, Sabrina Bernadez Pereira, Salvador Rassi, Sandrigo Mangini, Silvia Marinho Martins, Silvia Moreira Ayub Ferreira, Victor Sarli Issa.

\section{Contribuição dos autores}

Redação do manuscrito e Revisão crítica do manuscrito quanto ao conteúdo intelectual importante: Bocchi EA, Biolo A, Moura LZ, Figueiredo Neto JA, Montenegro CEL, Albuquerque DC

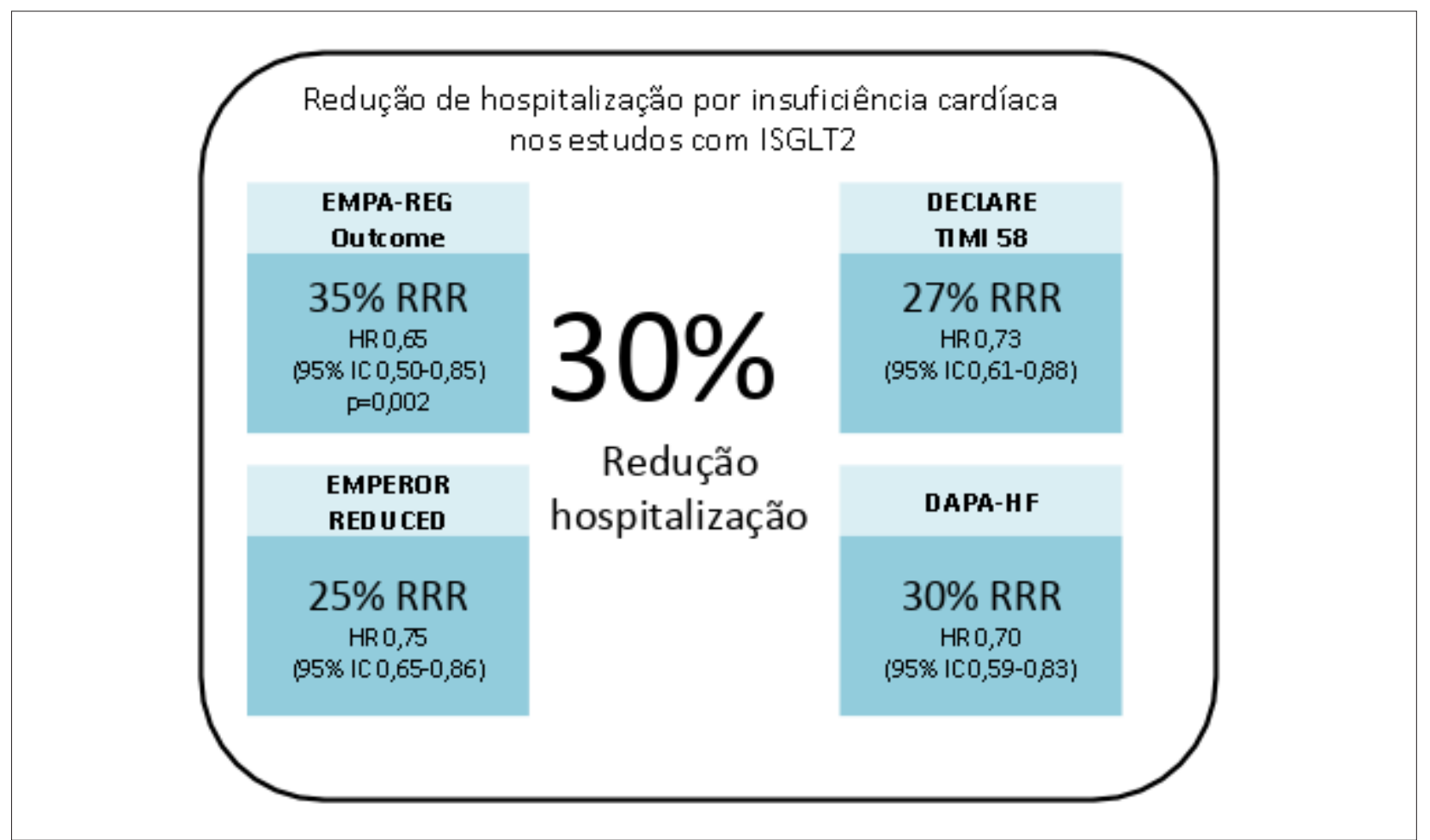

Figura 2 - Redução de hospitalização por insuficiência cardíaca nos estudos com ISGLT2. ISGLT2: inibidores do cotransportador de sódio-glicose-2; RRR: Redução do Risco Relativo; HR: hazard ratio; IC: intervalo de confiança. 


\section{Carta Científica}

\section{Potencial conflito de interesses}

Dr. Edimar Bocchi - Honorário de consultoria/palestra: Boehringer Ingelheim, Astra Zeneca. Verba de pesquisa: Astra Zeneca. Projetos de pesquisa: Boehringer Ingelheim, Astra Zeneca.

Dra. Lidia Zytynski Moura - Palestrante e do conselho consultivo da empresa Astra Zeneca. Zeneca.

Dr. Carlos Eduardo Lucena Montenegro - Palestrante da Astra

Dr. José Albuquerque de Figueiredo Neto - Palestrante da Astra Zeneca.

\section{Referências}

1. Verna, S., MCMurray, J.J.V. SGLT2 inhibitors and mechanisms of cardiovascular benefit: a state-of-the-art review. Diabetologia, 2018.

2. Lopaschuk, GD and Verma S. Mechanisms of Cardiovascular Benefits of Sodium Glucose Co-Transporter 2 (SGLT2) Inhibitors: A State-of-the-Art Review. JACC Basic Transl Sci, 2020; 5(6):632-644.

3. Fitchett D, Inzucchi SE, Cannon CP et al. Empagliflozin Reduced Mortality and Hospitalization for Heart Failure Across the Spectrum of Cardiovascular Risk in the EMPA-REG OUTCOME Trial. Circulation, 2019; 139(11): 1384-1395.

4. Wanner C, Lachin JM, Inzucchi SE et al (2018) Empagliflozin and clinical outcomes in patients with type 2 diabetes mellitus, established cardiovascular disease, and chronic kidney disease. Circulation $137: 119-129$.

5. Verma S, McMurray JJV, Cherney DZI (2017) The metabolodiuretic promise of sodium-dependent glucose cotransporter 2 inhibition: the search for the sweet spot in heart failure. JAMA Cardiol 2:939-940.

6. Sattar N, McLaren J, Kristensen SL et al. (2016) SGLT2 inhibition and cardiovascular events: why did EMPA-REG Outcomes surprise and what were the likely mechanisms? Diabetologia 59:1333-1339.

7. Ferrannini E, Mark M, Mayoux E (2016) CV protection in the EMPAREG OUTCOME Trial: a "Thrifty Substrate" hypothesis. Diabetes Care 39:1108-1114.

8. Baartscheer A, Schumacher CA, Wust RC et al. Empagliflozin decreases myocardial cytoplasmic $\mathrm{Na}+$ through inhibition of the cardiac $\mathrm{Na}+/ \mathrm{H}+$ exchanger in rats and rabbits. Diabetologia 2017; 60:568-573.

9. Fedak PW, Verma S, Weisel RD, Li RK. Cardiac remodeling and failure from molecules to man (part II). Cardiovasc Pathol 2005;14: 49-60.

10. Packer M. Do sodium-glucose co-transporter-2 inhibitors prevent heart failure with a preserved ejection fraction by counterbalancing the effects of leptin? A novel hypothesis. Diabetes Obes Metab. 2018; 20(6):1361-1366.

11. Packer M. Lessons learned from the DAPA-HF trial concerning the mechanisms of benefit of SGLT2 inhibitors on heart failure events in the context of other large-scale trials nearing completion. Cardiovasc Diabetol. 2019;18(1):129.

12. Zinman B, Wanner C, Lachin JM et al. for the EMPA-REG OUTCOME Investigators: Empagliflozin, Cardiovascular Outcomes, and Mortality in Type 2 Diabetes N Engl J Med 2015; 373:2117-2128

13. Neal B, Perkovic V, Mahaffey KW et al. CANVAS Program Collaborative Group. Canagliflozin and Cardiovascular and Renal Events in Type 2
Dr. Denilson Campos de Albuquerque - Pesquisador estudo Emperor. Conselho consultivo e palestra para Boehringer Ingelheim e Astra Zeneca.

\section{Fontes de financiamento}

O presente estudo não teve fontes de financiamento externas.

\section{Vinculação acadêmica}

Não há vinculação deste estudo a programas de pósgraduação.

Diabetes. N Engl J Med. 2017 Aug 17;377(7):644-657. doi: 10.1056/ NEJMoa1611925

14. Wiviott SD, Raz I, Bonaca MP et al. DECLARE-TIMI 58 Investigators. Dapagliflozin and Cardiovascular Outcomes in Type 2 Diabetes. N Engl J Med. 2019 Jan 24;380(4):347-357. doi: 10.1056/NEJMoa1812389

15. Cannon CP, Pratley R, Dagogo-Jack S et al. VERTIS C Investigators. Cardiovascular Outcomes with Ertugliflozin in Type 2 Diabetes. N Engl J Med. 2020 Oct 8;383(15):1425-1435. doi: 10.1056/NEJMoa2004967.

16. Ohkuma T, Komorita Y, Peters, SAE. et al. Diabetes as a risk factor for heart failure in women and men: a systematic review and meta-analysis of 47 cohorts including 12 million individuals. Diabetologia 62, 1550-1560 (2019).

17. Rawshani A, Rawshani A, Franzén S, et al. Risk Factors, Mortality, and Cardiovascular Outcomes in Patients with Type 2 Diabetes. N Engl J Med. 2018;379(7):633-644. doi:10.1056/NEJMoa1800256

18. Zelniker TA, Wiviott SD, Raz I, et al. SGLT2 inhibitors for primary and secondary prevention of cardiovascular and renal outcomes in type 2 diabetes: a systematic review and meta-analysis of cardiovascular outcome trials Lancet. 2019 Jan 5;393(10166):31-39. doi:10.1016/ S0140-6736(18)32590-X

19. Sinha B, Ghosal S. Meta-analyses of the effects of DPP-4 inhibitors, SGLT2 inhibitors and GLP1 receptor analogues on cardiovascular death, myocardial infarction, stroke and hospitalization for heart failure. Diabetes Res Clin Pract. 2019;150:8-16. doi:10.1016/j. diabres.2019.02.014

20. Packer M, Anker SD, Butler J, Bocchi E et al. EMPEROR-Reduced Trial Investigators. Cardiovascular and Renal Outcomes with Empagliflozin in Heart Failure. N Engl J Med. 2020 Oct 8;383(15):1413-1424. doi: 10.1056/NEJMoa2022190.

21. McMurray JJV, Solomon SD, Inzucchi SE et al DAPA-HF Trial Committees and Investigators. Dapagliflozin in Patients with Heart Failure and Reduced Ejection Fraction. N Engl J Med. 2019 Nov 21;381(21):19952008. doi: 10.1056/NEJMoa1911303

22. Perkovic V, Jardine MJ, Neal B, et al. CREDENCE Trial Investigators. Canagliflozin and Renal Outcomes in Type 2 Diabetes and Nephropathy. N Engl J Med. 2019 Jun 13;380(24):2295-2306. doi: 10.1056/ NEJMoa1811744.

23. Comitê Coordenador da Diretriz de Insuficiência Cardíaca, Rohde LEP, Montera MW, Bocchi EA, et al. Diretriz Brasileira de Insuficiência Cardíaca Crônica e Aguda. Arq Bras Cardiol. 2018 Sep;111(3):436-539 